J. Korean Math. Soc. 41 (2004), No. 5, pp. 913-920

\title{
ON THE RESIDUAL FINITENESS OF FUNDAMENTAL GROUPS OF GRAPHS OF CERTAIN GROUPS
}

\author{
GoANSU KiM
}

\begin{abstract}
We give a characterization for fundamental groups of graphs of groups amalgamating cyclic edge subgroups to be cyclic subgroup separable if each pair of edge subgroups has a non-trivial intersection. We show that fundamental groups of graphs of abelian groups amalgamating cyclic edge subgroups are cyclic subgroup separable, hence residually finite, if each edge subgroup is isolated in its containing vertex group.
\end{abstract}

\section{Introduction}

Let $\Gamma$ be a finite graph and let $Y$ be a maximal tree of $\Gamma$. The fundamental group $G$ of $\Gamma$ of groups $A_{v}$, amalgamating cyclic edge subgroups, is obtained by first taking a tree product $A$ of the $A_{v}$ according to $Y$ and then taking HNN extensions $G=\left\langle A, t_{1}, \ldots, t_{n}: t_{i}^{-1} h_{i} t_{i}=k_{i}, i=1, \ldots\right.$, $n\rangle$, where all the $h_{i}$ and $k_{i}$ are in the vertex groups $A_{v}$. Thus fundamental groups of graphs of groups are generalizations of amalgamated free products and HNN extensions of groups. Since the Baumslag-Solitar group $\left\langle a, t: t^{-1} a^{2} t=a^{3}\right\rangle$ is not residually finite [2], residual properties of HNN extensions or fundamental groups of graphs of groups are difficult to obtain. Recently, Raptis and Varsos [6,9] considered residual nilpotence and subgroup separability of fundamental groups of graphs of groups when the edge subgroups are of finite index in the containing vertex groups.

A group $G$ is cyclic subgroup separable (briefly, $\pi_{c}$ ) if, for each pair $g, x \in G$ such that $g \notin\langle x\rangle$, there exists a normal subgroup $N$ of finite index in $G$ (briefly, $N \triangleleft_{f} G$ ) such that $g \notin N\langle x\rangle$.

Received July 23, 2003.

2000 Mathematics Subject Classification: 20E26, 20E06, 20E08, 20F05.

Key words and phrases: residual finiteness, amalgamated free products, HNN extensions, tree products, fundamental groups of graphs of groups.

The author was partly supported by GARC-KOSEF and KOSEF 981-0101-001-2. 
In this paper, using a characterization (Theorem 2.3 below) for HNN extensions to be $\pi_{c}$, we consider the cyclic subgroup separability and residual finiteness of fundamental groups of graphs of groups when the edge subgroups are infinite cyclic.

We construct a fundamental group of a bouquet of two cycles which is not residually finite (Example 2.6) if each pair of edge subgroups has the trivial intersection. However, we give a characterization for fundamental groups of graphs of groups amalgamating cyclic edge subgroups to be $\pi_{c}$ if each pair of edge subgroups has a non-trivial intersection (Corollary 3.2). On the other hand, we also show that fundamental groups of any graph of abelian groups amalgamating cyclic edge subgroups are cyclic subgroup separable, hence residually finite, if each edge subgroup is isolated in its containing vertex group (Theorem 3.7).

\section{Preliminaries}

Let $\Gamma=(V, E)$ be a graph, where $V$ is a set of vertices and $E$ is a set of edges. To each vertex $v$ in $V$, we assign a group $G_{v}$. To each edge $e$ in $E$, we assign a group $G_{e}$ and monomorphisms $\alpha_{e}$ and $\beta_{e}$ embedding $G_{e}$ to the vertex groups at the end of $e$. Then, for a maximal tree $T$ of $\Gamma$, the fundamental group of the graph $(\Gamma)$ of groups $G_{v}$ amalgamating the edge subgroups is defined to be the group generated by the generators and relations of the vertex groups and additional generators $t_{e}$ for each $e \in E$ with the additional relations $t_{e}^{-1}\left(g_{e} \alpha_{e}\right) t_{e}=g_{e} \beta_{e}$ for each $g_{e} \in G_{e}$ where $t_{e}=1$ if $e$ is an edge of $T$. Each of $G_{e} \alpha_{e}$ and $G_{e} \beta_{e}$ is called an edge subgroup in its containing vertex group. It is well-known that the fundamental group of a graph of groups is independent from the choice of the maximal tree [7]. In particular, if the graph $\Gamma$ is a tree then the fundamental group of $\Gamma$ of groups $A_{v}$ is called a tree product (see [4]) of the $A_{v}$.

Throughout this paper we consider fundamental groups of finite graphs of groups amalgamating infinite cyclic edge subgroups. If the graph $\Gamma$ is not connected, then the fundamental group of $\Gamma$ is a free product of fundamental groups of the connected subgraphs of $\Gamma$. Since free products of residually finite or $\pi_{c}$ groups are again residually finite or $\pi_{c}$ [1], we only consider the case that the graphs are connected.

We begin with tree products of groups.

THEOREM 2.1. Tree products of finitely generated free-by-finite or polycyclic-by-finite groups amalgamating cyclic subgroups are $\pi_{c}$, hence residually finite. 
Proof. It was known by $[8,3]$ that finitely generated free-by-finite or polycyclic-by-finite groups are weak potent, equivalently regular quotients, and $\pi_{c}$. Thus the result follows from Theorem 3.4 in [3].

As we mentioned in the beginning of the introduction, a fundamental group of a graph of groups amalgamating cyclic edge subgroups is presented by

$$
G=\left\langle A, t_{1}, \ldots, t_{n}: t_{i}^{-1} h_{i} t_{i}=k_{i}, i=1, \ldots, n\right\rangle
$$

where $A$ is a tree product for a maximal tree of the graph and where $h_{i}, k_{i}$ are in the vertex groups $A_{v}$ of $A$. Thus, we need to study the residual property of HNN extensions of groups. The following results in [5] are useful for this purpose.

Definition 2.2. Let $A$ be a group and let $h, k \in A$ be of infinite order. Then $A$ is said to be quasi-regular at $\{h, k\}$ if, for each given integer $\epsilon>0$, there exist an integer $\lambda_{\epsilon}>0$ and $N_{\epsilon} \triangleleft f A$, depending on $\epsilon$, such that $N_{\epsilon} \cap\langle h\rangle=\left\langle h^{\epsilon \lambda_{\epsilon}}\right\rangle$ and $N_{\epsilon} \cap\langle k\rangle=\left\langle k^{\epsilon \lambda_{\epsilon}}\right\rangle$.

Theorem 2.3. [5] Let $A$ be $\pi_{c}$ and let $h, k \in A$ be of infinite order. Then the HNN extension $\left\langle A, t: t^{-1} h t=k\right\rangle$ is $\pi_{c}$ if and only if $A$ is quasiregular at $\{h, k\}$.

For a finitely generated abelian group $A$, it is not difficult to see that $A$ is quasi-regular at $\{h, k\}$ if and only if $\langle h\rangle \cap\langle k\rangle=1$ or $h^{s}=k^{ \pm s}$ for some $s$. Using this, we have the followings:

THEOREM 2.4. [5] Let $A$ be a finitely generated abelian group. Let $h, k \in A$ be of infinite order. The HNN extension $G=\left\langle A, t: t^{-1} h t=k\right\rangle$ is $\pi_{c}$ if and only if $\langle h\rangle \cap\langle k\rangle=1$ or $h^{s}=k^{ \pm s}$ for some $s>0$.

THEOREM 2.5. [5] Let $A$ be a finitely generated abelian group. Let $h, k \in A$ be of infinite order. The HNN extension $G=\left\langle A, t: t^{-1} h t=k\right\rangle$ is residually finite if and only if one of the followings holds:

(i) If $A=\langle b\rangle, h=b^{\alpha}$ and $k=b^{\beta}$, then $|\alpha|=1$ or $|\beta|=1$ or $\alpha \pm \beta=0$.

(ii) If $A$ is not cyclic then $\langle h\rangle \cap\langle k\rangle=1$ or $h^{s}=k^{ \pm s}$ for some $s>0$.

The above two results characterized the cyclic subgroup separability and residual finiteness of fundamental groups of a graph of an abelian group if the graph has a vertex and an edge. But if a graph has a vertex and two edges, a bouquet with two cycles, then as in the next example, the fundamental group may not be even residually finite.

EXAMPLE 2.6. Let $G=\left\langle A, t_{1}, t_{2}: t_{1}^{-1} a t_{1}=b, t_{2}^{-1} a^{2} b^{3} t_{2}=a^{2} b^{6}\right\rangle$, where $A=\langle a, b:[a, b]=1\rangle$. Suppose $G$ is residually finite. Let $g=$ 
$\left[t_{2} a b^{3} t_{2}^{-1}, b\right]$. Then $g \neq 1$. Since $G$ is residually finite, there exists $N \triangleleft_{f} G$ such that $g \notin N$ and $a \notin N$. Let $|\bar{a}|=\alpha$ and $\left|\bar{a} \bar{b}^{3}\right|=\beta$ in $\bar{G}=G / N$. Since $a \sim_{G} b,|\bar{b}|=\alpha$. Hence $\beta \mid \alpha$. Since $a^{2} b^{3} \sim_{G} a^{2} b^{6}$ and $\left|\bar{a} \bar{b}^{3}\right|=\beta$, we have $\bar{a}^{2 \beta} \bar{b}^{6 \beta}=1=\bar{a}^{2 \beta} \bar{b}^{3 \beta}$. Thus $\bar{b}^{3 \beta}=1$ and $\bar{a}^{2 \beta}=1$. Since $a \sim_{G} b, \bar{b}^{2 \beta}=1$. Hence $\bar{b}^{\beta}=1$. Thus $\alpha \mid \beta$. Therefore $\alpha=\beta$. Now, since $\bar{g}=\left[\bar{t}_{2} a b^{3} t_{2}^{-1}, \bar{b}\right] \neq 1$, we have $\bar{a} \bar{b}^{3} \notin\left\langle\bar{a}^{2} \bar{b}^{6}\right\rangle=\left\langle\left(\bar{a} \bar{b}^{3}\right)^{2}\right\rangle$. This implies $2|\beta=| \bar{a} \bar{b}^{3} \mid$. Let $\beta=\alpha=2 \alpha_{1}$. Then $\left(\bar{a}^{2} \bar{b}^{6}\right)^{\alpha_{1}}=\bar{a}^{\alpha} \bar{b}^{3 \alpha}=1$. Hence $\left(\bar{a}^{2} \bar{b}^{3}\right)^{\alpha_{1}}=1$, since $a^{2} b^{3} \sim_{G} a^{2} b^{6}$. Then $\bar{a}^{2 \alpha_{1}} \bar{b}^{3 \alpha_{1}}=1=\bar{a}^{2 \alpha_{1}} \bar{b}^{6 \alpha_{1}}$. Thus $\bar{b}^{3 \alpha_{1}}=1=\bar{a}^{2 \alpha_{1}}$ and hence $\bar{b}^{2 \alpha_{1}}=1$. Therefore $\bar{b}^{\alpha_{1}}=1$, hence $\alpha \mid \alpha_{1}$, a contradiction. Thus $G$ is not residually finite.

We note that, in the above example, $G$ is a fundamental group of a bouquet of two cycles and all pairs of edge subgroups have the trivial intersection and the edge subgroup $\left\langle a^{2} b^{6}\right\rangle$ is not isolated in the vertex group $A$. Thus, in the next section, we consider fundamental groups of graph of groups if the graph has one closed cycle, or if each pair of two edge subgroups has nontrivial intersections, or if each edge subgroup is isolated in its containing vertex group.

\section{Main results}

TheOREM 3.1. Let $A$ be $\pi_{c}$ and let $h_{i}, k_{i} \in A$ be of infinite order such that $\left\langle h_{i}\right\rangle \cap\left\langle k_{i}\right\rangle \neq 1$ for each $i=1, \ldots, n$. Then $G_{n}=$ $\left\langle A, t_{1}, \ldots, t_{n}: t_{i}^{-1} h_{i} t_{i}=k_{i}, i=1, \ldots, n\right\rangle$ is $\pi_{c}$ if and only if, for each $i=1, \ldots, n, h_{i}^{s_{i}}=k_{i}^{ \pm s_{i}}$ for some $s_{i}>0$.

Proof. We first show that $G_{1}=\left\langle A, t_{1}: t_{1}^{-1} h_{1} t_{1}=k_{1}\right\rangle$ is $\pi_{c}$ if and only if $h_{1}^{s_{1}}=k_{1}^{ \pm s_{1}}$ for some $s_{1}>0$, whenever $\left\langle h_{1}\right\rangle \cap\left\langle k_{1}\right\rangle \neq 1$. If $G_{1}$ is $\pi_{c}$ then, by Theorem 2.3, $A$ is quasi-regular at $\left\{h_{1}, k_{1}\right\}$. Hence $h_{1}^{s_{1}}=k_{1}^{ \pm s_{1}}$ for some $s_{1}>0$, since $\left\langle h_{1}\right\rangle \cap\left\langle k_{1}\right\rangle \neq 1$. Conversely, suppose $h_{1}^{s_{1}}=k_{1}^{ \pm s_{1}}$ for some $s_{1}>0$. Let $\epsilon$ be a given integer. Since $\left|h_{1}\right|,\left|k_{1}\right|$ are infinite, $h_{1}^{i}, k_{1}^{i} \notin\left\langle h_{1}^{s_{1} \epsilon}\right\rangle=\left\langle k_{1}^{s_{1} \epsilon}\right\rangle$ for all $1 \leq i<s_{1} \epsilon$. Now $A$ is $\pi_{c}$. This implies that there exists $N_{1} \triangleleft_{f} A$ such that $h_{1}^{i}, k_{1}^{i} \notin N_{1}\left\langle h_{1}^{s_{1} \epsilon}\right\rangle$ for all $1 \leq i<s_{1} \epsilon$. Hence $N_{1} \cap\left\langle h_{1}\right\rangle=\left\langle h_{1}^{s_{1} \epsilon \delta_{1}}\right\rangle$ for some $\delta_{1}>0$ and $N_{1} \cap\left\langle k_{1}\right\rangle=\left\langle k_{1}^{s_{1} \in \delta_{2}}\right\rangle$ for some $\delta_{2}>0$. Then $\left\langle h_{1}^{s_{1}} \in \delta_{1}\right\rangle=N_{1} \cap\left\langle h_{1}^{s_{1}}\right\rangle=N_{1} \cap\left\langle k_{1}^{s_{1}}\right\rangle=\left\langle k_{1}^{s_{1}} \in \delta_{2}\right\rangle$. Hence $\delta_{1}=\delta_{2}$. Therefore, $N_{1} \cap\left\langle h_{1}\right\rangle=\left\langle h_{1}^{s_{1} \in \delta_{1}}\right\rangle$ and $N_{1} \cap\left\langle k_{1}\right\rangle=\left\langle k_{1}^{s_{1} \in \delta_{1}}\right\rangle$ for some $\delta_{1}>0$. Thus $A$ is quasi-regular at $\left\{h_{1}, k_{1}\right\}$. Hence $G_{1}$ is $\pi_{c}$ by Theorem 2.3. 
Suppose $G_{n}$ is $\pi_{c}$. Since $\left\langle A, t_{i}: t_{i}^{-1} h_{i} t_{i}=k_{i}\right\rangle$ is a subgroup of $G_{n}$, it must be $\pi_{c}$. Hence, by above, $h_{i}^{s_{i}}=k_{i}^{ \pm s_{i}}$ for some $s_{i}>0$.

Conversely, suppose, for each $i=1, \ldots, n, h_{i}^{s_{i}}=k_{i}^{ \pm s_{i}}$ for some $s_{i}>0$. Then, by above, $G_{1}$ is $\pi_{c}$. Inductively, we assume $G_{n-1}=$ $\left\langle A, t_{1}, \ldots, t_{n-1}: t_{i}^{-1} h_{i} t_{i}=k_{i}, i=1, \ldots, n-1\right\rangle$ is $\pi_{c}$. Then, as above, $G_{n-1}$ is quasi-regular at $\left\{h_{n}, k_{n}\right\}$. Hence $G_{n}$ is $\pi_{c}$ by Theorem 2.3.

In Lemma 2.5 of [5], it was shown that $G_{1}$ above is $\pi_{c}$ if and only if $h_{1}^{s_{1}}=k_{1}^{ \pm s_{1}}$ for some $s_{1}>0$ with the additional condition that $A$ is weak $\left\langle h_{1}\right\rangle$-potent.

COROLlary 3.2. Let $A_{v}$ be finitely generated free-by-finite or polycyclic-by-finite groups. Let $G$ be a fundamental group of a graph of the $A_{v}$, amalgamating cyclic edge subgroups, presented by $G=\left\langle A, t_{1}, \ldots, t_{n}\right.$ $\left.: t_{i}^{-1} h_{i} t_{i}=k_{i}, i=1, \ldots, n\right\rangle$, where $A$ is a tree product of the $A_{v}$ according to a maximal tree of the graph and where $\left\langle h_{i}\right\rangle \cap\left\langle k_{i}\right\rangle \neq 1$ for each $i=1, \ldots, n$. Then $G$ is $\pi_{c}$ if and only if, for each $i=1, \ldots, n, h_{i}^{s_{i}}=k_{i}^{ \pm s_{i}}$ for some $s_{i}>0$.

Proof. The result follows from Theorem 3.1 and Theorem 2.1.

Note that, in the above corollary, $h_{i}$ and $k_{i}$ may not be in the same vertex group.

Corollary 3.3. Let $A$ be finitely generated free-by-finite or polycyclic-by-finite groups. Let $h_{i}, k_{i} \in A$ be of infinite order such that $h_{i}^{s_{i}}=$ $k_{i}^{ \pm s_{i}}$ for some $s_{i}>0$. Then $G=\left\langle A, t_{1}, \ldots, t_{n}: t_{i}^{-1} h_{i} t_{i}=k_{i}, i=1, \ldots, n\right\rangle$ is $\pi_{c}$, hence, residually finite.

If a graph $\Gamma$ has one cycle, a loop or a closed path, then the fundamental group of $\Gamma$ of groups amalgamating cyclic edge subgroups is presented by $G=\left\langle A, t: t^{-1} h t=k\right\rangle$, where $A$ is a tree product of a maximal tree of $\Gamma$.

TheOREM 3.4. Let $A_{i}$ be polycyclic-by-finite groups. Let $\Gamma$ be a graph with one cycle. Let $G$ be a fundamental group of $\Gamma$ of the $A_{i}$ amalgamating cyclic edge subgroups, where each edge subgroup is contained in the center of its containing vertex group. If each pair $\langle h\rangle,\langle k\rangle$ of edge subgroups has the property $\langle h\rangle \cap\langle k\rangle=1$ or $h^{s}=k^{ \pm s}$ for some $s$, then $G$ is $\pi_{c}$, hence residually finite.

Proof. Let $G=\left\langle A, t: t^{-1} h t=k\right\rangle$, where $A$ is a tree product of a maximal tree $Y$ of $\Gamma$ and where $h, k$ are in the centers of their containing vertex groups of the tree product $A$. First of all, $A$ is $\pi_{c}$ by Theorem 2.1. 
Hence, if $h^{s}=k^{ \pm s}$ for some $s$, then $G$ is $\pi_{c}$ by Theorem 3.1. So, we suppose that $\langle h\rangle \cap\langle k\rangle=1$. To apply Theorem 2.3, we shall show that $A$ is quasi-regular at $\{h, k\}$. In fact, we can show that, for any $\alpha, \beta$, there exists $N \triangleleft_{f} A$, depending on $\alpha, \beta$, such that $N \cap\langle h\rangle=\left\langle h^{\alpha}\right\rangle$ and $N \cap\langle k\rangle=\left\langle k^{\beta}\right\rangle$. For this, we use an induction on the number of vertex groups of the tree product $A$.

If $A$ has one vertex, then $h, k \in Z(A)$. Since $\bar{A}=A /\left\langle h^{\alpha}\right\rangle\left\langle k^{\beta}\right\rangle$ is again a polycyclic-by-finite group, $\bar{A}$ is residually finite. Hence there exists $\bar{N} \triangleleft_{f} \bar{A}$ such that $\bar{N} \cap\langle\bar{h}\rangle=1$ and $\bar{N} \cap\langle\bar{k}\rangle=1$. Let $N$ be the preimage of $\bar{N}$ in $A$. Then $N \cap\langle h\rangle=\left\langle h^{\alpha}\right\rangle$ and $N \cap\langle k\rangle=\left\langle k^{\beta}\right\rangle$, as required.

Let $A=B{ }_{\langle c\rangle} A_{v}$, where $v$ is an extreme vertex of the maximal tree $Y$ and $B$ is the tree product of the subtree $Y-\{v\}$. For an induction, we suppose if, for each pair $\langle a\rangle,\langle b\rangle$ of edge subgroups of $B,\langle a\rangle \cap\langle b\rangle=1$ then there exists $M \triangleleft_{f} B$ such that $M \cap\langle a\rangle=\left\langle a^{\alpha}\right\rangle$ and $M \cap\langle b\rangle=\left\langle b^{\beta}\right\rangle$ for any $\alpha, \beta$.

(i) Suppose $h, k \in B$ (or similarly $h, k \in A_{v}$ ). By induction hypothesis, there exists $M \triangleleft_{f} B$ such that $M \cap\langle h\rangle=\left\langle h^{\alpha}\right\rangle$ and $M \cap\langle k\rangle=\left\langle k^{\beta}\right\rangle$ for any $\alpha, \beta$. Let $M \cap\langle c\rangle=\left\langle c^{s}\right\rangle$. Since $c \in Z\left(A_{v}\right)$, we have a homomorphic image $\bar{A}=B / M *_{\langle\bar{c}\rangle} A_{v} /\left\langle c^{s}\right\rangle$ of $A$. Since $\bar{A}$ is residually finite, as before, there exists $\bar{N} \triangleleft_{f} \bar{A}$ such that $\bar{N} \cap\langle\bar{h}\rangle=1$ and $\bar{N} \cap\langle\bar{k}\rangle=1$. Let $N$ be the preimage of $\bar{N}$ in $A$. Then $N \cap\langle h\rangle=\left\langle h^{\alpha}\right\rangle$ and $N \cap\langle k\rangle=\left\langle k^{\beta}\right\rangle$, as required.

(ii) Suppose $h \in B$ and $k \in A_{\psi}$. Since $\langle h\rangle \cap\langle k\rangle=1,\langle h\rangle \cap\langle c\rangle=1$ or $\langle k\rangle \cap\langle c\rangle=1$. Suppose $\langle h\rangle \cap\langle c\rangle=1$. If $\langle k\rangle \cap\langle c\rangle \neq 1$, then let $\left\langle k^{\beta}\right\rangle \cap\langle c\rangle=\left\langle c^{s}\right\rangle$. Since $\langle h\rangle \cap\langle c\rangle=1$, by induction hypothesis there exists $M \triangleleft_{f} B$ such that $M \cap\langle h\rangle=\left\langle h^{\alpha}\right\rangle$ and $M \cap\langle c\rangle=\left\langle c^{s}\right\rangle$. Thus we have a homomorphic image $\bar{A}=B / M *_{\langle\bar{c}\rangle} A_{v} /\left\langle k^{\beta}\right\rangle$ of $A$. On the other hand, if $\langle k\rangle \cap\langle c\rangle=1$, then we have a homomorphic image $\bar{A}=$ $B / M *_{\langle\bar{c}\rangle} A_{v} /\left\langle k^{\beta}\right\rangle\left\langle c^{s}\right\rangle$ of $A$. In both cases, since $\bar{A}$ is residually finite, there exists $\bar{N} \triangleleft_{f} \bar{A}$ such that $\bar{N} \cap\langle\bar{h}\rangle=1$ and $\bar{N} \cap\langle\bar{k}\rangle=1$. Let $N$ be the preimage of $\bar{N}$ in $A$. Then $N \cap\langle h\rangle=\left\langle h^{\alpha}\right\rangle$ and $N \cap\langle k\rangle=\left\langle k^{\beta}\right\rangle$, as required.

Therefore $A$ is quasi-regular at $\{h, k\}$, hence $G$ is $\pi_{c}$ by Theorem 2.3 .

Corollary 3.5. Let $\Gamma$ be a graph with one cycle. Let $G$ be a fundamental group of $\Gamma$ of finitely generated abelian groups $A_{i}$ amalgamating cyclic edge subgroups. If each pair $\langle h\rangle,\langle k\rangle$ of edge subgroups has the 
property $\langle h\rangle \cap\langle k\rangle=1$ or $h^{s}=k^{ \pm s}$ for some $s$, then $G$ is $\pi_{c}$, hence residually finite.

We note that the conditions, $\langle h\rangle \cap\langle k\rangle=1$ or $h^{s}=k^{ \pm s}$ for some $s$, are best possible, since the Baumslag-Solitar group $\left\langle a, t: t^{-1} a^{2} t=a^{3}\right\rangle$ is not residually finite and the group $\left\langle a, t: t^{-1} a t=a^{2}\right\rangle$ is residually finite by Theorem 2.5 .

Lemma 3.6. Let $A$ be $\pi_{c}$ and let $h_{i}, k_{i} \in A$ be of infinite order. Suppose, for each $\epsilon>0$, there exist $\lambda$ and $N_{\epsilon} \triangleleft_{f} A$ such that $N_{\epsilon} \cap\left\langle h_{i}\right\rangle=$ $\left\langle h_{i}^{\lambda \epsilon}\right\rangle$ and $N_{\epsilon} \cap\left\langle k_{i}\right\rangle=\left\langle k_{i}^{\lambda \epsilon}\right\rangle$ for each $i$. Then $G_{n}=\left\langle A, t_{1}, \ldots, t_{n}\right.$ : $\left.t_{i}^{-1} h_{i} t_{i}=k_{i}, i=1, \ldots, n\right\rangle$ is $\pi_{c}$.

Proof. Since $A$ is quasi-regular at $\left\{h_{1}, k_{1}\right\}, G_{1}=\left\langle A, t_{1}: t_{1}^{-1} h_{1} t_{1}=k_{1}\right\rangle$ is $\pi_{c}$ by Theorem 2.3. Inductively, suppose

$$
G_{n-1}=\left\langle A, t_{1}, \ldots, t_{n-1}: t_{i}^{-1} h_{i} t_{i}=k_{i}, i=1, \ldots, n-1\right\rangle
$$

is $\pi_{c}$. Then, by assumption, for each $\epsilon>0$ there exist $\lambda$ and $N_{\epsilon} \triangleleft_{f} A$ such that, in $\bar{A}=A / N_{\epsilon},\left|\bar{h}_{i}\right|=\epsilon \lambda=\left|\bar{k}_{i}\right|$ for each $1 \leq i \leq n$. Hence, there exists a homomorphism $\pi: G_{n-1} \rightarrow \bar{G}_{n-1}$, where $\bar{G}_{n-1}=\left\langle\bar{A}, t_{1}, \ldots, t_{n-1}\right.$ : $\left.t_{i}^{-1} \bar{h}_{i} t_{i}=\bar{k}_{i}, i=1, \ldots, n-1\right\rangle$ and $\bar{A}=A / N_{\epsilon}$. Since $\bar{A}$ and $\left|\bar{h}_{i}\right|=\left|\bar{k}_{i}\right|$ are finite, $\bar{G}_{n-1}$ is $\pi_{c}$ by [1]. Hence $\bar{G}_{n-1}$ is residually finite. Thus there exists $\bar{M} \triangleleft_{f} \bar{G}_{n-1}$ such that $\bar{M} \cap\left\langle\bar{h}_{n}\right\rangle=1=\bar{M} \cap\left\langle\bar{k}_{n}\right\rangle$. Let $M=\pi^{-1}(\bar{M})$. Then $M \triangleleft_{f} G_{n-1}, M \cap\left\langle h_{n}\right\rangle=\left\langle h_{n}^{\epsilon \lambda}\right\rangle$ and $M \cap\left\langle k_{n}\right\rangle=\left\langle k_{n}^{\epsilon \lambda}\right\rangle$. Thus $G_{n-1}$ is quasi-regular at $\left\{h_{n}, k_{n}\right\}$. Hence $G_{n}$ is $\pi_{c}$ by Theorem 2.3.

Recall that a subgroup $H$ of $G$ is said to be isolated in $G$ if, for each $a \in G$ and $n \neq 0, a^{n} \in H$ implies $a \in H$.

THEOREM 3.7. Let $G$ be a fundamental group of any graph of finitely generated abelian groups $A_{i}$ amalgamating isolated cyclic edge subgroups. Then $G$ is $\pi_{c}$, hence, residually finite.

Proof. Let $G=\left\langle A, t_{1}, \ldots, t_{n}: t_{i}^{-1} h_{i} t_{i}=k_{i}, i=1, \ldots, n\right\rangle$, where $A$ is a tree product of the $A_{i}$ according to a maximal tree of the graph and $\left\langle h_{i}\right\rangle,\left\langle k_{i}\right\rangle$ are isolated in their respective vertex groups. We note that if $\langle h\rangle$ is isolated in a finitely generated abelian group $A_{s}$ then $A_{s}^{\epsilon} \cap\langle h\rangle=$ $\left\langle h^{\epsilon}\right\rangle$ for each $\epsilon$. Thus we have a homomorphism $\pi: A \rightarrow \bar{A}$, where $\bar{A}$ is a tree product of the $\bar{A}_{s}=A_{s} / A_{s}^{\epsilon}$, since all edge subgroups are isolated in their respective vertex groups. Then $\left|\bar{h}_{i}\right|=\epsilon=\left|\bar{k}_{i}\right|$ in $\bar{A}$. Since $\bar{A}$ is $\pi_{c}$ by Theorem 2.1 , hence residually finite, there exists $\bar{N} \triangleleft_{f} \bar{A}$ such that $\bar{N} \cap\left\langle\bar{h}_{i}\right\rangle=1=\bar{N} \cap\left\langle\bar{k}_{i}\right\rangle$ for each $i=1, \ldots, n$. Let $N=\pi^{-1}(\bar{N})$. Then $N \triangleleft_{f} A, N \cap\left\langle h_{i}\right\rangle=\left\langle h_{i}^{\epsilon}\right\rangle$ and $N \cap\left\langle k_{i}\right\rangle=\left\langle k_{i}^{\epsilon}\right\rangle$ for each $i=1, \ldots, n$. Since $A$ is $\pi_{c}$, it follows from Lemma 3.6 that $G$ is $\pi_{c}$. 
We note that, in Example 2.6, the edge subgroup $\left\langle a^{2} b^{6}\right\rangle$ is not isolated in the free abelian group $A=\langle a, b\rangle$, and the resulting group $G$ is not residually finite.

\section{References}

[1] R. B. J. T. Allenby and R. J. Gregorac, On locally extended residually finite groups, Lecture Notes in Math. 319 (1973), 9-17, Springer Verlag, New York.

[2] G. Baumslag and D. Solitar, Some two-generator one-relator non-Hopfian groups, Bull. Amer. Math. Soc. 38 (1962), 199-201.

[3] B. Evans, Cyclic amalgamations of residually finite groups, Pacific J. Math. 55 (1974), 371-379.

[4] G. Kim and C. Y. Tang, A criterion for the conjugacy separability of amalgamated free products of conjugacy separable groups, J. Algebra 184 (1996), 1052-1072.

[5] Cyclic subgroup separability of $H N N$-extensions with cyclic associated subgroups, Canad. Math. Bull. 42 (1999),335-343.

[6] E. Raptis and D. Varsos, On subgroup separability of the fundamental group of a finite graph of groups, Demonstatio Math. 29 (1996), 43-52.

[7] J. P. Serre, Trees, Springer-Verlag, Berlin-Heidelberg-New York, 1980.

[8] C. Y. Tang, Conjugacy separability of generalized free products of certain conjugacy separable groups, Canad. Math. Bull. 38 (1995), no. 1, 120-127.

[9] D. Varsos, The residual nilpotence of the fundamental group of certain graphs of groups, Houston J. Math. 22 (1996), 233-248.

Department of Mathematics

Yeungnam University

Kyongsan 712-749, Korea

E-mail: gskim@ynucc.yeungnam.ac.kr 